\title{
EVALUATION OF THE APPLICABILITY OF ULTRASONIC VELOCITY PROFILING IN CONDITIONS RELATED TO WET LOW INTENSITY MAGNETIC SEPARATION
}

\author{
Jan F. Stener ${ }^{* 1}$, Johan E. Carlson ${ }^{2}$, Bertil I. Pålsson ${ }^{1}$ and Anders Sand ${ }^{1}$ \\ ${ }^{1}$ Minerals and Metals Research Laboratory \\ Dept. of Civil, Environmental and Natural Resources Engineering \\ Luleå University of Technology \\ SE-971 87 Luleå, SWEDEN \\ ${ }^{2}$ Div. of Systems and Interaction \\ Dept. of Computer Science, Electrical and Space Engineering \\ Luleå University of Technology \\ SE-971 87 Luleå, SWEDEN \\ *Jan.Stener@ltu.se
}

Keywords: Iron ores, Magnetic separation, Process instrumentation.

\begin{abstract}
The internal material transport and selection processes of the wet low-intensity magnetic separators (LIMS) are poorly understood; this calls for improved measurement techniques. In this work an ultrasonic velocity profiling (UVP) technique for measuring how material flow speed varies with penetration depth is presented. A measurement depth of just a couple of centimetres would greatly improve our understanding of the separation process in a LIMS.
\end{abstract}

When applied to flows of mineral suspensions with high volumetric solids concentration, similar to those in the separators, UVP is unique in combining:

- Non-intrusive measurements.

- Operates using just one sensor element (transducer). 
- Relatively good spatial resolution.

- Penetrates opaque suspensions.

- Fast sampling rate.

Here, flows are studied in a rectangular duct $(50 \times 75 \mathrm{~mm})$. Using magnetite suspensions, measurement through the whole depth of $50 \mathrm{~mm}$ is made with good accuracy. Velocity profiles are presented for solids concentrations of 5 and $9 \%$ solids by volume. Even at $9 \mathrm{vol} \%$ solids it is possible to reach a penetration depth of more than $25 \mathrm{~mm}$.

\section{INTRODUCTION}

The wet low-intensity magnetic separator (LIMS) is the workhorse for winning of fine ferromagnetic particles from ore pulps; despite this the internal workings of the machine are poorly understood. However, as experienced by the industry, when pressed to higher capacities and with higher concentrate quality demands, it has started to show some limitations. In particular, fairly coarse gangue minerals have a tendency to be locked into the magnetic concentrate despite being fully liberated. To increase the understanding of this problem the use of computer simulations were attempted by Lejon Isaksson (2008). One conclusion from that work was that trustworthy simulations need measurements for validation. This became the starting point for the work presented here. The maximum depth of a full size separator tank is about $100 \mathrm{~mm}$, but to verify simulations a measurement depth of just a couple of centimetres would suffice, and this is the goal of our work.

\subsection{Magnetic separation}

The wet LIMS (Fig. 1) consists of a rotating non-magnetic drum with a number of internally fixed magnets arranged with alternating polarity. This rotating shell is partially submerged in a tank into which the ground magnetite ore suspension is fed. The magnetic portion of the feed material is attracted towards the drum surface and then carried through the alternating magnetic 
field and out through the concentrate discharge. The history and physics of wet LIMS is described in more detail by Parker (1977).

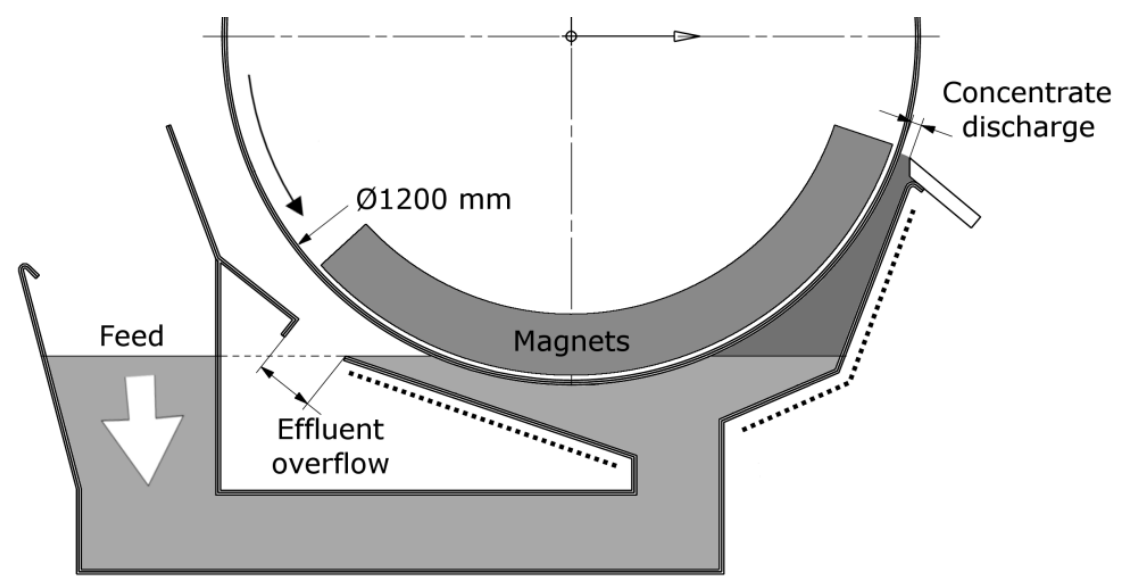

Fig. 1. Cross section of wet LIMS of counter-current type (design by Metso, www.metso.com). Dotted lines mark walls of special interest for mounting of sensors for flow velocity measurements.

The amount of research done on wet LIMS is limited, but some work can be found; for example Lantto (1977a and 1977b) investigated how various factors, such as the number of separation stages, magnetic field design, tank design, pulp density and magnetic flocculation, influence the performance of wet LIMS for a titaniferrous magnetite ore and found that magnetic flocculation had central significance.

Rayner and Napier-Munn (2003) combines empirical advice and experimental trials to develop process models for wet drum magnetic separators. Also here magnetic flocculation has a central role; a model to predict loss of magnetic material is presented where the loss depends on a first order flocculation rate and the residence time in the separation zone. Even though this work is aimed at wet drum magnetic separators used for dense medium recovery much of the theory should also be applicable to magnetite concentrations.

Recently Dworzanowski (2010) described, from a general point of view, how the different design and operating variables interact and how they affect performance and also provides 
guidelines on operation. Factors given high importance include tank design, magnet assembly configuration, feed preparation, feed rate, level control and drum/tank distance.

Generally one of the challenges of optimizing LIMS is the balance between attracting too much material, and thus getting mixed grains of low grade in the concentrate, and attracting too little material and losing the very fine (liberated) magnetic fraction to the tailings.

To gain a deeper understanding of the process of wet LIMS, measurements of internal particle flow are needed. To obtain good quality data the following specification need to be met:

1. The sensor is required to operate from one direction only, this since the separator design allows physical access to the flow from only one side (dashed lines in Fig. 1).

2. A technique capable of penetrating opaque and attenuating suspension is required.

3. Some degree of spatial resolution is needed to interpret the results.

4. A non-intrusive technique is preferred since the best results are obtained if the flow is not disturbed and also the internal environment could be very harsh on equipment.

5. Information about both flow speed and suspension concentration variations is desired.

\subsection{Ultrasonic measurement methods}

To meet the specifications listed above an ultrasonic method was chosen. Ultrasonic techniques are (almost) non-invasive quantitative techniques capable of operating in opaque fluids. There are three main categories of ultrasonic flow velocity measurement methods; transit time, Doppler-based and speckle correlation techniques. Most of these methods originate from the field of medicine or navigation but have in more recent years found many other applications. Hein and O'Brien (1993) have made a review summarising this development.

In transit-time flow measurement systems two ultrasonic transducers operate by alternately transmitting and receiving bursts of sound energy between them. The difference in measured 
transit time is directly proportional to the velocity of the liquid in the pipe. Transit-time techniques measure the bulk flow velocity.

Ultrasonic Doppler flow meters employ the frequency shift (Doppler Effect) of an ultrasonic signal when it is reflected by suspended particles or gas bubbles in motion. Doppler based methods measure the motion of the particles. This technique is used by for example Takeda (1986), Wiklund et al. (2008), Chemloul et al. (2009) and Hunter et al. (2011); a summary of results comparable to those in this paper can be found in Table 3.

Speckle correlation techniques track the movement of particles or, as in the current case, local density variations in a suspension. (A visualisation of particle distribution in turbulent flow of suspension can be found in Wood et al., 2005). Short ultrasonic pulses are generated and in turn create backscatter signals which are run through a cross-correlation process to extract a time delay. From this delay the particle displacement and velocity can be calculated. This technique is computationally demanding, but as the generally available computing power increase this limitation rapidly diminishes. Already in 1976 Dotti et al. used an ultrasonic cross-correlation technique to measure blood flow.

In the current work a single transducer is used to acquire an 1D profile of the flow, but it is also possible to use an array of sensors to acquire a 2D profile; cf. Sandrin et al. (2001), Manneville et al. (2001) and Carlson and Ing (2002). Bamberger and Greenwood (2004) and Furlan et al. (2012) also used ultrasonic methods to measure slurry density and solids concentration.

\section{METHOD}

To develop a method capable of measuring in the demanding environment of a wet LIMS tank a variation of the speckle correlation technique was selected. The method, here called ultrasonic velocity profiling (UVP), uses an ultrasound transducer to track particle motion in the flow. The transducer first transmits a short pulse and is then used as a receiver to record the backscattered 
signal (echo). This backscattered signal contains information about local concentration variations.

By acquiring two backscatter signals closely spaced in time and then cross-correlating them it is possible to follow how groups of particles move. By dividing the backscatter signals into short segments and cross-correlating them piecewise it is possible to get information about how this movement varies with position. The distance travelled by a group of particles are related to the time delay (lag) corresponding to the strongest correlation. Since the time between the two backscatter signals is known it is possible to calculate particle velocity. The transducer inclination angle $(\theta)$ is used to project the measurements on the direction of flow. The method is described in more detail in the signal processing section.

During signal processing it is assumed that the volume responsible for measured backscatters at each time, the interaction volume (IV), has a volume of negligible size. In reality the volume is finite; Jorgensen et al. (1973) describe the IV as flat drop shape, but here it is sufficient to treat the IV as a flat cylinder, see Fig. 2. Close to the transducer the diameter of the cylinder $(d)$ is the same as the transducer diameter and then the diameter increases as the pulse diverges (with divergence angle $\alpha$ ). The height $(h)$ of the cylinder is related to the pulse duration and the speed of sound. Note that the interaction volume is a theoretical concept, in reality there is no distinctly defined border between affected and unaffected suspension. 


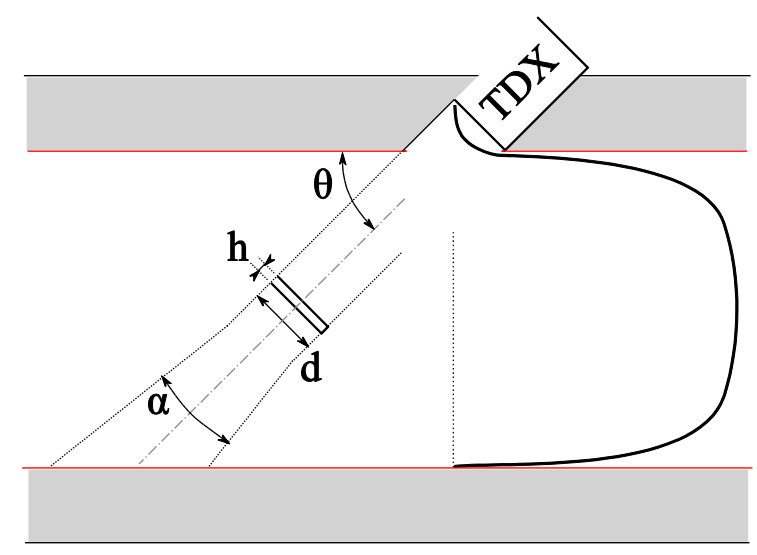

Fig. 2. Ultrasonic pulse path and the concept of interaction volume. Wall thickness, distance and transducer (TDX) are approximately to scale. A typical turbulent flow velocity profile is included to the right.

Due to the finite volume of the IV velocity profiles measured using UVP can become distorted close to walls. With the current setup approximately $5 \mathrm{~mm}$ from each wall is affected. For the top wall (transducer side) this means that the flow might be underestimated since the IV is only partly in the flow. For the bottom side this means that a signal reflected twice from the bottom wall influences the measurement. To some extent it would be possible to compensate for these effects during signal processing, but this is not done here.

\subsection{Experimental setup}

The proposed method is developed and evaluated using a laboratory-scale experimental setup. The experimental setup (Fig. 3) consists of a closed loop made of rubber hosing with an internal diameter of $50 \mathrm{~mm}$. The flow is driven by a progressing cavity pump (Netzsch NM053BY01L06B, www.netzsch.com) capable of supplying up to $14 \mathrm{~m}^{3} / \mathrm{h}$, corresponding to an average flow rate of up to $1 \mathrm{~m} / \mathrm{s}$ in the measurement section. The pump motor is controlled with an AC drive (Emotron VFX48-010, www.emotron.com) and the suspension temperature is stabilised by a heat exchanger fed with cold tap water. 


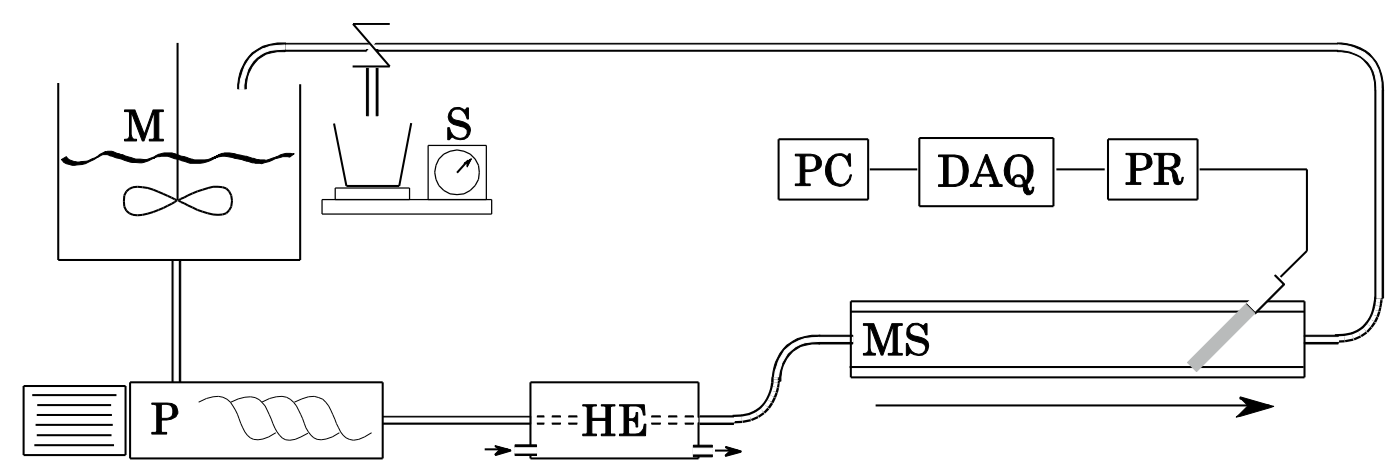

Fig. 3. Experimental setup comprising mixer (M), pump (P), heat exchanger (HE), measurement section (MS). Also sampling position ( $\mathrm{S}$ ) and data acquisition equipment is shown; pulser-receiver (PR), digitizer (DAQ) and computer (PC).

The walls of the measurement section (MS) (depth $0.050 \mathrm{~m}$, width $0.075 \mathrm{~m}$ and length $1 \mathrm{~m}$ ) are made from $12 \mathrm{~mm}$ clear polycarbonate and the ultrasonic transducer is fitted at a $45^{\circ}$ inclination in one end of the section. The length of straight duct in front of the transducer is $0.85 \mathrm{~m}$, corresponding to 14 hydraulic diameters. To evacuate entrained air from the volume closest to the transducer a small peristaltic pump (Watson-Marlow 503S, www.watson-marlow.com) is used. The flow rate (through a $\varnothing 1.5 \mathrm{~mm}$ hole) is negligible compared to the main flow.

\subsection{Material and suspension properties}

The magnetite material used was supplied by LKAB (www.lkab.com) from the KA1 concentrator at Kiruna, Sweden. It was sampled from the feed stream to the second stage of wet LIMS (overflow hydrocyclone). The material density, $5.0 \mathrm{~kg} / \mathrm{dm}^{3}$, is measured with a pycnometer (Micromeritics 1305, www.micromeritics.com). Particle size analysis by laser diffraction (Cilas 1064, www.cilas.com) showed that the mean diameter is $34 \mu \mathrm{m}$ and mechanical sieving showed that approx. $85 \mathrm{wt} \%$ of the material is smaller than $45 \mu \mathrm{m}$.

The dry magnetite powder is mixed with water to reach desired concentrations. The stated concentrations are checked by sampling the stream, at (S) in Fig. 3, and then drying the sample and calculating solids content. In the presented measurements, the solids content was about 
$4.7 \%$ and $9.0 \%$ by volume, corresponding to $20 \%$ and $33 \%$ by mass, respectively. To prevent sedimentation a high flow rate is used during all experiments.

\subsection{Electronic equipment}

The measurements are made using the immersion transducers in Table 1. These are controlled by a computer controlled pulser-receiver (Panametrics 5800PR, www.olympus-ims.com). The received backscatter signal is processed by a digitizer (ADQ214, www.spdevices.com) featuring $400 \mathrm{MS} / \mathrm{s}$ at 14 bit resolution. Before each ultrasonic measurement the suspension temperature in the mixer (M) is measured using a digital thermometer (ASL F250 MKII, www.aslltd.co.uk).

Table 1. Ultrasonic transducers (Olympus, www.olympus-ims.com).

\begin{tabular}{|l|l|l|l|l|}
\hline $\begin{array}{l}\text { Manufacture r-specified } \\
\text { centre frequency }\end{array}$ & $\begin{array}{l}\text { Approximate } \\
\text { wavelength }\end{array}$ & $\begin{array}{l}\text { Divergence } \\
\text { half angle }\end{array}$ & Near field & Part No. \\
\hline $1.0 \mathrm{MHz}$ & $1.50 \mathrm{~mm}$ & $8.1^{\circ}$ & $27 \mathrm{~mm}$ & $\mathrm{~V} 303$ \\
\hline $2.25 \mathrm{MHz}$ & $0.67 \mathrm{~mm}$ & $3.6^{\circ}$ & $61 \mathrm{~mm}$ & $\mathrm{~V} 306$ \\
\hline $3.5 \mathrm{MHz}$ & $0.43 \mathrm{~mm}$ & $2.3^{\circ}$ & $94 \mathrm{~mm}$ & $\mathrm{~V} 382$ \\
\hline $5.0 \mathrm{MHz}$ & $0.30 \mathrm{~mm}$ & $1.6^{\circ}$ & $135 \mathrm{~mm}$ & $\mathrm{~V} 309$ \\
\hline
\end{tabular}


Table 2. Experimental parameters used.

\begin{tabular}{|l|l|l|l|}
\hline Property & Settings I* & Settings II ** & Equation \\
\hline Vertical measurement duct depth & $50 \mathrm{~mm}$ & $50 \mathrm{~mm}$ & \\
\hline Transducer inclination angle $(\theta)$ & $45^{\circ}$ & $45^{\circ}$ & \\
\hline Transducer nominal diameter $(d)$ & $\varnothing 13 \mathrm{~mm}$ & $\varnothing 13 \mathrm{~mm}$ & \\
\hline Sample frequency $\left(f_{s}\right)$ & $400 \mathrm{MHz}$ & $200 \mathrm{MHz}$ & \\
\hline Pulse repetition frequency $\left(1 / T_{P R F}\right)$ & $5 \mathrm{kHz}$ & $8 \mathrm{kHz}$ & \\
\hline Measurements per flow profile $(O)$ & 200 & 200 & \\
\hline Window length $(M)$ & 1000 & 1000 & Eq. 2 \\
\hline Vertical spatial resolution $(\Delta y)$ & $2.7 \mu \mathrm{m}$ & $5.3 \mu \mathrm{m}$ & Eq. 5 \\
\hline Horizontal velocity resolution $(\Delta v)$ & $13 \mathrm{~mm} / \mathrm{s}$ & $42 \mathrm{~mm} / \mathrm{s}$ & \\
\hline Sound velocity in suspension $(c) * * *$ & $1480-1510 \mathrm{~m} / \mathrm{s}$ & $1480-1510 \mathrm{~m} / \mathrm{s}$ & \\
\hline
\end{tabular}

* Used in Fig. 5a-b. ** Used in Fig. 5c-f. *** Depending on suspension temperature.

\subsection{Signal processing}

Signal acquisition control and signal processing is carried out in MATLAB (www.mathworks.com) running custom code. Assume that two backscatter signals (echoes), $p_{1}[n]$ and $p_{2}[n]$ are acquired by sampling the received analogue signals at a sampling rate $T_{\mathrm{s}}=1 / f_{\mathrm{s}}$ so that

$p_{i} n=p_{1} n T_{s}, i=1,2, n=1,2, \ldots, N$.

The vertical spatial distance between each sample $p_{i}[n]$ is calculated using the vertical spatial resolution

$\Delta y=\frac{T_{S} c}{2 \cos \theta}$

where $c$ is the speed of sound in the medium (calculated using measured temperature and standard data for water). $\theta$ is the angle between the ultrasonic transducer and the direction of 
flow (see Fig. 2) and the factor 2 is introduced since the pulse travels back and forth through the suspension.

The two signals are acquired at a pulse-repetition frequency (PRF) so that the time between the two acquisitions is $T_{\mathrm{PRF}}=1 / \mathrm{PRF}$. If the $\mathrm{PRF}$ is sufficiently high, we can assume that the particles in the interaction volume (IV) has translated like an entity, so that the backscatter signatures differ only by a time delay $\left(n_{0}\right)$ proportional to the movement of the suspension during the time $T_{\mathrm{PRF}}$

The time for a pulse to travel from the transducer to a particular IV and back varies only with the position of the IV, this means that each sample (n) will carry information from different depth in the flow. By dividing the sampled signal into short segments

$\mathbf{x}_{1}[k]=\left\{p_{1}[1+k], p_{1}[2+k], \ldots, p_{1}[M+k]\right\}$

$\mathbf{x}_{2}[k]=\left\{p_{2}[1+k], p_{2}[2+k], \ldots, p_{2}[M+k]\right\}$

the displacement of the flow can be calculated as a function of depth. The windows are $M$ samples long taken from $p_{1}[n]$ and $p_{2}[n]$, respectively, and window index $k=0,1, \ldots, N-M-1$.

The first step towards finding this time delay between each of these windows is to do a cross correlation of the two windows

$X C[m, k]={ }_{\mathrm{k}=0}^{N-M-1} p_{1} n p_{2} n-m$,

where $m$ is a sufficiently large span of lags; e.g. $m=-256,-255, \ldots, 0, \ldots, 255,256$.

The horizontal flow speed corresponding to a certain lag $m$ is calculated using the horizontal flow velocity resolution

$\Delta v=\frac{T_{s} c}{2 \sin \theta} \cdot \frac{1}{T_{P R F}}$

Fig. 4a shows the cross-correlation $X C$ as a function of windows index converted to depth (using Eq. 2) and lags converted to flow velocity (using Eq. 5). The final particle flow velocity 
profile in Fig. $4 \mathrm{~b}$ has been estimated by finding the flow speed corresponding to the maximum correlation.
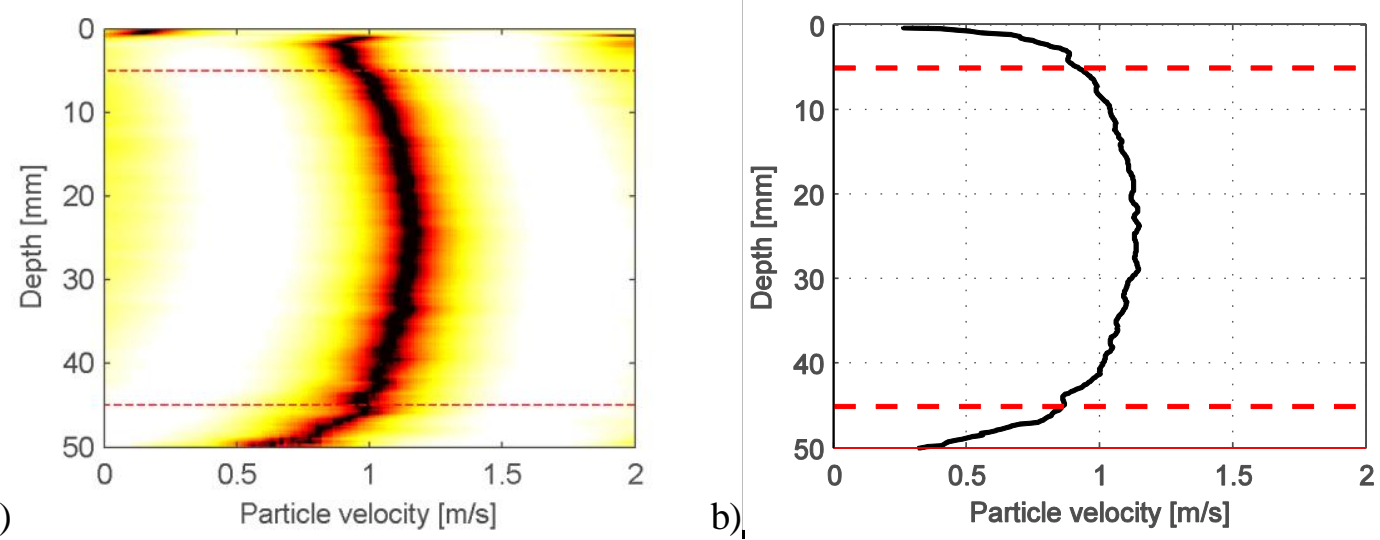

Fig. 4a. Cross-correlation strength, colouring indicates how the correlation strength between the two windows $\left(\mathbf{x}_{1}\right.$ and $\mathbf{x}_{2}$ ) varies with lag $(m)$ and vertical position. Dark colours indicate strong correlation. Fig. 4b. Corresponding flow velocity profile calculated as the velocity corresponding to the strongest correlation at each depth.

To obtain better estimates of the particle velocity profiles, the above procedure is repeated $O=200$ times, each resulting in an estimated velocity profile. For each depth the median flow velocity is calculated; the median is known to be good at suppressing outliers, meaning that this should also efficiently discard estimates where the two signals have decorrelated. After median filtering all flow profiles have been filtered with a moving average smoothing filter

$v_{s} k=\frac{1}{2 N+1} v k-\frac{N}{2}+v k-\frac{N}{2}+1+\cdots+v k+\frac{N}{2}$. 


\section{RESULTS}

At solids concentrations below 2 vol\% the method is capable of measurements through the whole available depth of $50 \mathrm{~mm}$. In Fig. 5a-d, where a solids concentration of 4.7 vol\% is used, the $2.25 \mathrm{MHz}$ transducer produces the most reliable results. When the transmitting frequency is increased the signal is attenuated faster and does not penetrate the full $50 \mathrm{~mm}$. The penetration depth was about $25 \mathrm{~mm}$ for the $3.5 \mathrm{MHz}$ and $20 \mathrm{~mm}$ for the $5 \mathrm{MHz}$ transducer. With the $1 \mathrm{MHz}$ transducer a problem of using too low frequency is shown; this long wavelength $(1.5 \mathrm{~mm})$ does not create enough contrast for the cross-correlation process to produce an equally good result.

When the concentration is increased to $9 \mathrm{vol} \%$, in Fig. 5e-f, the $2.25 \mathrm{MHz}$ transducer still produce the most reliable results and it is possible to reach a depth of almost $30 \mathrm{~mm}$. 
a) 5 vol\% solid, $1 \mathrm{MHz}$ transducer.

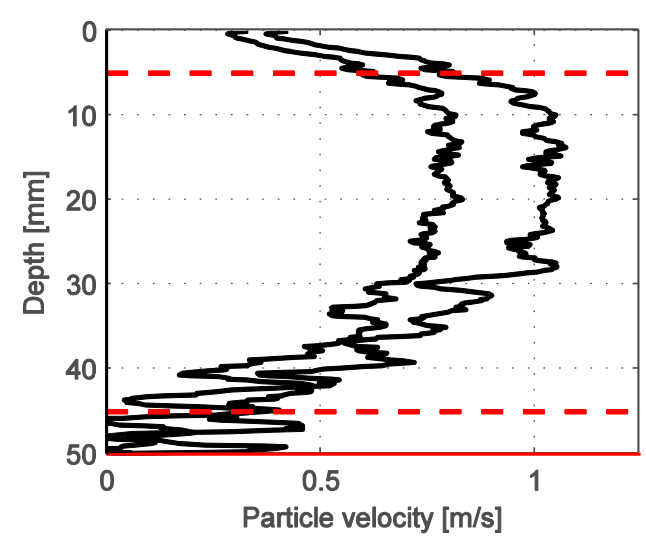

c) 5 vol $\%$ solid, $3.5 \mathrm{MHz}$ transducer.

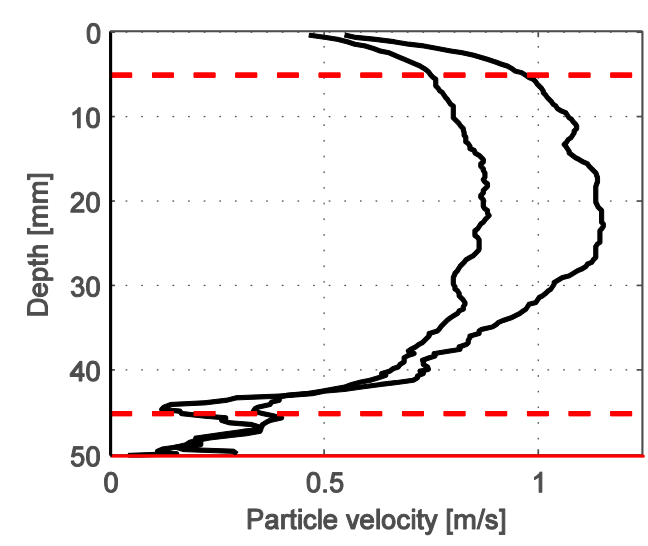

e) 9 vol\% solid, $1 \mathrm{MHz}$ transducer.

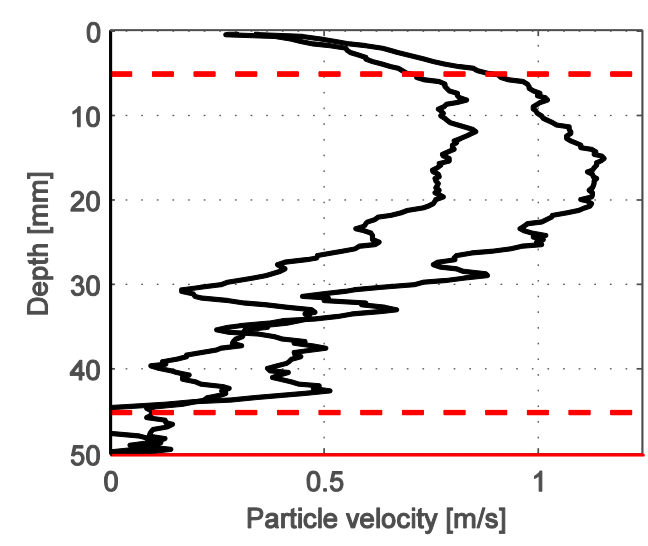

b) 5 vol\% solid, $2.25 \mathrm{MHz}$ transducer.

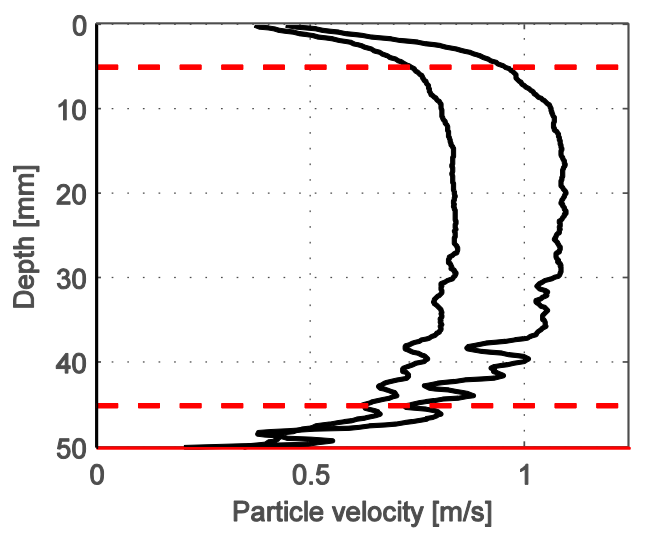

d) 5 vol\% solid, $5 \mathrm{MHz}$ transducer.

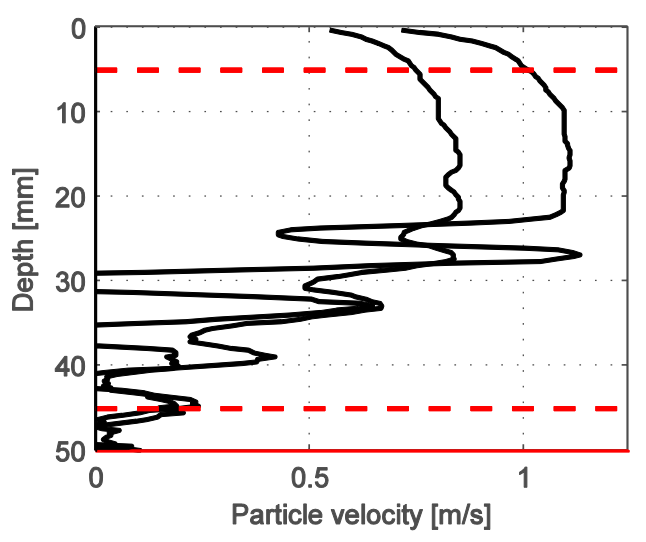

f) 9 vol\% solid, $2.25 \mathrm{MHz}$ transducer.

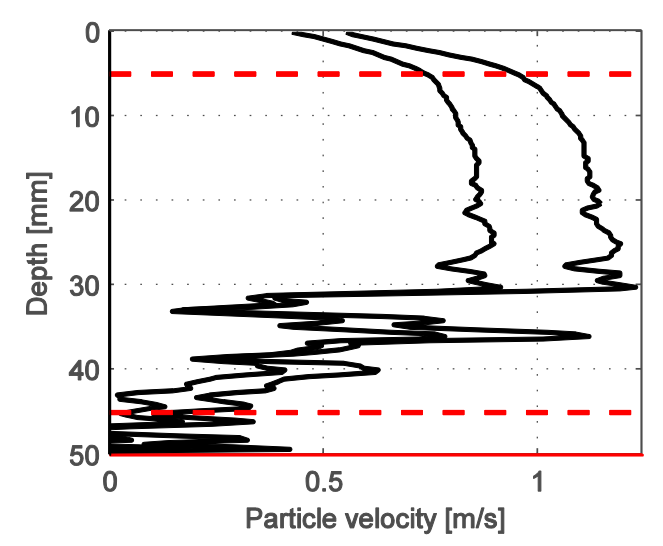

Fig. 5. Measurements on flow of magnetite suspension at bulk flow speed of 0.75 and $1 \mathrm{~m} / \mathrm{s}$. Two different concentrations and four different transducers are compared. The transducer is placed above the figures. 0 and $50 \mathrm{~mm}$ on the vertical axis represent the top respective bottom of the measurement duct. The dashed (red) lines mark areas where wall effects might influence the measurements. 


\section{DISCUSSION}

The penetration depth of an ultrasonic pulse is mainly dependent upon ultrasound frequency (quadratic dependency), particle radius (cubic dependency) and mass fraction solids (linear dependency) (Carlson, 2010). If these results are compared with those in the literature (Table 3) both deeper measurements, and also measurements in thicker suspensions can be found, but not both at the same time. Also some of these measurements use transducers on two opposite sides of the flow, which cuts the ultrasonic path length in half.

Table 3. Ultrasonic flow profiles in literature.

\begin{tabular}{|l|l|l|l|l|l|l|}
\hline Refe rence & $\begin{array}{l}\text { Solid } \\
\text { Material }\end{array}$ & $\begin{array}{l}\text { Avg. } \\
\text { particle } \\
\text { dia. }\end{array}$ & $\begin{array}{l}\text { Max. } \\
\text { solids } \\
\text { conc. }\end{array}$ & $\begin{array}{l}\text { Max. penetration } \\
\text { depth } \\
\text { (transducer } \\
\text { inclination angle) }\end{array}$ & $\begin{array}{l}\text { Trans ducer } \\
\text { centre } \\
\text { frequency }\end{array}$ & $\begin{array}{l}\text { Transducer } \\
\text { size }\end{array}$ \\
\hline $\begin{array}{l}\text { Takeda } \\
(1986)\end{array}$ & $\mathrm{Al}_{2} \mathrm{O}_{3}$ & $5 \mu \mathrm{m}$ & Low & $\varnothing 12 \mathrm{~mm}\left(60^{\circ}\right)$ & $4.2 \mathrm{MHz}$ & $\varnothing 4 \mathrm{~mm}$ \\
\hline $\begin{array}{l}\text { Carlson } \\
\text { et al. (2002) }\end{array}$ & $\begin{array}{l}\text { Magne- } \\
\text { tite }\end{array}$ & $54 \mu \mathrm{m}$ & Low & $\varnothing 34 \mathrm{~mm}\left(45^{\circ}\right)$ & $3.5 \mathrm{MHz}$ & $0.4 \mathrm{~mm} * *$ \\
\hline $\begin{array}{l}\text { Wiklund } \\
\text { et al. (2008) }\end{array}$ & Mineral & $11-90$ & $20 \mathrm{wt} \%$ & $\varnothing 36 \mathrm{~mm}\left(\sim 70^{\circ}\right)$ & $2-4 \mathrm{MHz}$ & $\varnothing 5,10 \mathrm{~mm}$ \\
\hline $\begin{array}{l}\text { Chemloul } \\
\text { et al. (2009) }\end{array}$ & Glass & $270 \mu \mathrm{m}$ & $2 \mathrm{vol} \%$ & $\varnothing 20 \mathrm{~mm}\left(67^{\circ}\right)$ & $8 \mathrm{MHz}$ & $\varnothing 2 \mathrm{~mm}$ \\
\hline $\begin{array}{l}\text { Hunter } \\
\text { et al. (2011) }\end{array}$ & Glass & $10 \mu \mathrm{m}$ & $5 \mathrm{wt} \%$ & $300 \mathrm{~mm}\left(0^{\circ} *\right)$ & $1 \mathrm{MHz}$ & $\varnothing 15 \mathrm{~mm}$ \\
\hline $\begin{array}{l}\text { Kotzé } \\
\text { et al. (2013) }\end{array}$ & Kaolin & $\mathrm{N} / \mathrm{A}$ & $\begin{array}{l}17 \\
\text { vol\% }\end{array}$ & $\varnothing 16 \mathrm{~mm}\left(70^{\circ}\right)$ & $4 \mathrm{MHz}$ & $\varnothing 8 \mathrm{~mm}$ \\
\hline
\end{tabular}

* Sedimenting particles. ** Pitch of 64 element transducer.

The ultrasonic transducer centre frequency can be selected for each application to reach optimal performance. Also the vertical penetration depth can be increased by increasing the transducer inclination angle $(\theta)$, though this will be done with the expense of reduced measurement accuracy and robustness; using an inclination angle of $70^{\circ}$ instead of $45^{\circ}$ could increase the 
penetration depth up to $32 \%$. Another thing to consider is the main excitation of the transducer. In the current setup the pulser-reciever generates a negative impulse $(-300 \mathrm{~V})$ with pulse energy of $100 \mu \mathrm{J}$. According to specification the transducers used can handle stronger excitation, which could yield improved penetration depth.

As a comparison, and check-up on the measurements, basic Computational Fluid Dynamics (CFD) simulations have been carried out in Comsol Multiphysics 4.3 (www.comsol.com), cf. Fig. 6. The model consists of a duct with the same dimensions as the measurement duct where water $\left(\right.$ at $20^{\circ} \mathrm{C}$ ) flows with an average velocity of $1.0 \mathrm{~m} / \mathrm{s}$. The software generated "physics controlled" mesh consists of $535 \cdot 10^{3}$ elements. The $k-\varepsilon$ turbulence model is used and all other settings are left at their defaults.
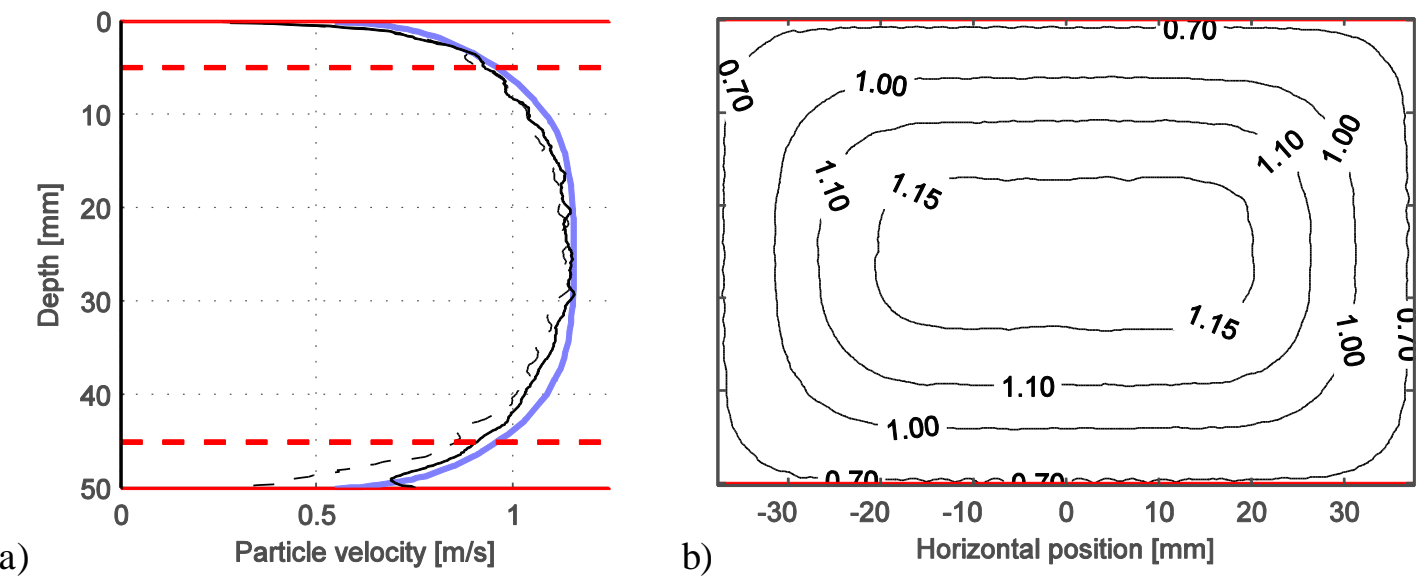

Fig. 6a. Measured particle velocity profiles (bulk flow speed $1 \mathrm{~m} / \mathrm{s}$ ) where suspensions with low solid content is used; $<0.5 \mathrm{vol} \%$ solids (- -) and approximately $2 \mathrm{vol} \%$ solids (-). These are compared to a CFD simulation (-). Fig. 6b. 2D cross-section from CFD simulation showing velocity contours $[\mathrm{m} / \mathrm{s}]$.

Apart from the interaction volume being affected by the walls, also the actual flow is to some extent affected by the recess close to the ultrasonic transducer. The principal effect on the flow profile is included close to the transducer (TDX) in Fig. 2. Here this effect is assumed to have negligible effect on the final flow profiles, especially since the profiles are already distorted close to the walls due to effects of the finite interaction volume. The comparison indicates that 
the measured velocity profiles closely follow a turbulent flow profile over the whole depth of the duct.

Another phenomenon affecting the measurements is the estimation of the speed of sound in the suspension. In Fig. 5 the speed of sound is assumed to be the same as for water of the same temperature. The speed of sound in water at $20^{\circ} \mathrm{C}$ and $30^{\circ} \mathrm{C}$ is $1482 \mathrm{~m} / \mathrm{s}$ respective $1509 \mathrm{~m} / \mathrm{s}$. But the speed of sound also depends on solids concentration and particle distribution. For example Wiklund (2008) uses a value of $1530 \mathrm{~m} / \mathrm{s}$ for mineral slurry at $20^{\circ} \mathrm{C}$. Since the measured flow speed depends linearly on the estimation of speed of sound the practical effect is small; for instance, the error resulting from using $1482 \mathrm{~m} / \mathrm{s}$ instead of $1530 \mathrm{~m} / \mathrm{s}$ is only $3 \%$.

The portion of the flow being considered in each velocity calculation depends heavily on the actual shape of the interaction volume. The strongest backscatters have the most influence on the measurements, but particles from a span in depth of up to, and even more than, $11 \mathrm{~mm}$ could influence one single point on the velocity profile. Also the time to complete 200 repeated measurements is in the order of $100 \mathrm{~ms}$. In these experiments a turbulent flow is required to keep the dense particles in suspension. This basically means that, because of turbulent vortices, on a small length scale the fluid flow can have any direction. This makes it difficult to differentiate between measurement fluctuations caused by vortices and fluctuations being measurement error.

However it is possible to get good qualitative measurements in demanding environments and since just one sensor is needed setup and signal acquisition can be simple. Also no calibration is needed; measured velocity depends only on the speed of sound in the suspension. As shown here, the technique can operate up to at least 9 vol\% solids concentration and down to very dilute suspensions; some small amount of solid particles is required to create backscatters though. 


\section{CONCLUSIONS}

The penetration depth depends strongly on the volume fraction of solid material, but to be able to measure particle flow velocity only a short distance inside a process unit could yield a much deeper understanding of the behaviour of the LIMS unit process.

Under the conditions created in these experiments the $2.25 \mathrm{MHz}$ transducer produce the most reliable results. At 2, 5 and 9 vol\% solids concentration the vertical penetration depth is 50,35 and $25 \mathrm{~mm}$ respectively.

This technique has great potential in the field of minerals engineering, wherever there is a need to measure particle flow in narrow channels.

\section{ACKNOWLEDGEMENT}

Financial support to the project WET LIMS - Measurements and models, by the Hjalmar Lundbohm Research Centre (HLRC), is gratefully acknowledged. Practical support from LKAB personnel in plant sampling is greatly appreciated.

\section{REFERENCES}

Bamberger, J. A., \& Greenwood, M. S. (2004). Measuring fluid and slurry density and solids concentration non-invasively. Ultrasonics, 42(1-9), 563-567. doi: 10.1016/j.ultras.2004.01.032

Carlson, J., \& Ing, R. K. (2002). Ultrasonic particle velocimetry in multiphase flows. IEEE Ultrasonics Symposium, 761-764. doi: 10.1109/ULTSYM.2002.1193510

Carlson, J. E. (2010). Estimation of particle size distributions in solid/liquid suspensions based on wide-band ultrasound attenuation measurements. Ultrasonics Symposium(IUS), 2010 IEEE, 707-710. 
Chemloul, N. S., Chaib, K., \& Mostefa, K. (2009). Simultaneous measurements of the solid particles velocity and concentration profiles in two phase flow by pulsed ultrasonic doppler velocimetry. Journal of the Brazilian Society of Mechanical Sciences and Engineering, 31 (4), 333-343.

Dworzanowski, M. (2010). Optimizing the performance of wet drum magnetic separators. The Journal of the Southern African Institute of Mining and Metallurgy, 110, 643-653.

Furlan, J. M., Mundla, V., Kadambi, J., Hoyt, N., Visintainer, R., \& Addie, G. (2012). Development of A-scan ultrasound technique for measuring local particle concentration in slurry flows. Powder Technology, 215-216, 174-184. doi: 10.1016/j.powtec.2011.09.044

Hein, I. A., \& O'Brien, W. D. (1993). Current time-domain methods for assessing tissue motion by analysis from reflected ultrasound echoes-a review. IEEE Transactions on Ultrasonics, Ferroelectrics and Frequency Control, 40(2), 84-102. doi: 10.1109/58.212556

Hunter, T. N., Peakall, J., \& Biggs, S. R. (2011). Ultrasonic velocimetry for the in situ characterisation of particulate settling and sedimentation. Minerals Engineering, 24(5), 416423. doi: $10.1016 /$ j.mineng.2010.12.003

Jorgensen, J. E., Campau, D. N., \& Baker, D. W. (1973). Physical characteristics and mathematical modelling of the pulsed ultrasonic flowmeter. Medical and Biological Engineering, 11(4), 404-421. doi: 10.1007/BF02483686

Karmazin, V. V., Bikbov, M. A., \& Bikbov, A. A. (2002). The energy saving technology of beneficiation of iron ore. Magnetic and Electrical Separation, 11(4), 211-224. doi: $10.1080 / 1055691021000062813$ 
Kotzé, R., Wiklund, J., \& Haldenwang, R. (2013). Optimisation of pulsed ultrasonic velocimetry system and transducer technology for industrial applications. Ultrasonics, 53(2), 459-469. doi: 10.1016/j.ultras.2012.08.014

Lantto, H. (1977). Effect of magnetic flocculation in the beneficiation of magnetic materials. Acta Polytechnica Scandinavia, 133

Lantto, H. (1977). Factors affecting low-intensity wet magnetic separation. Acta Polytechnica Scandinavica, Chemistry Including Metallurgy Series, 135

Lejon Isaksson, L. (2008). Våta svagmagnetiska separatorer - modeller och funktion. (Master thesis, Luleå University of technology).

Manneville, S., Sandrin, L., \& Fink, M. (2001). Investigating a stretched vortex with ultrafast two-dimensional ultrasonic speckle velocimetry. Physics of Fluids, 13(6), 1683-1690. doi: $10.1063 / 1.1370388$

Parker, M. R. (1977). The physics of magnetic separation. Contemporary Physics, 18(3), 279306. doi: $10.1080 / 00107517708231486$

Rayner, J. G., \& Napier-Munn, T. J. (2003). A mathematical model of recovery of dense medium magnetics in the wet drum magnetic separator. International Journal of Mineral Processing, 69(1-4), 157-173. doi: 10.1016/S0301-7516(02)00122-9

Sandrin, L., Manneville, S., \& Fink, M. (2001). Ultrafast two-dimensional ultrasonic speckle velocimetry: A tool in flow imaging. Applied Physics Letters, 78(8), 1155-1157. doi: $10.1063 / 1.1350622$ 
Takeda, Y. (1986). Velocity profile measurement by ultrasound doppler shift method. International Journal of Heat and Fluid Flow, 7(4), 313-318.

doi: $10.1016 / 0142-727 X(86) 90011-1$

Wiklund, J., \& Stading, M. (2008). Application of in-line ultrasound doppler-based UVP-PD rheometry method to concentrated model and industrial suspensions. Flow Measurement and Instrumentation, 19(3-4), 171-179. doi: 10.1016/j.flowmeasinst.2007.11.002

Wood, A. M., Hwang, W., \& Eaton, J. K. (2005). Preferential concentration of particles in homogeneous and isotropic turbulence. International Journal of Multiphase Flow, 31(10-11), 1220-1230. doi: 10.1016/j.jmultiphaseflow.2005.07.001 